\title{
HILBERT'S TWELFTH PROBLEM AND $L$-SERIES
}

\author{
BY H. M. STARK ${ }^{1}$
}

Communicated by Olga Taussky Todd, March 14, 1977

Let $k$ be a totally real number field of degree $n \geqslant 2$ with conjugate fields $k=k^{(1)}, \ldots, k^{(n)}$. Let $I(F)$ denote the group of fractional ideals of $k$ generated by those integral ideals relatively prime to a given integral ideal, $F$. Let $S(F)$ denote the subgroup of $I(F)$ generated by those principal integral ideals $(\alpha)$ with $\alpha \equiv 1(\bmod F)$. The quotient group $H=I(F) / S(F)$ is the ray class group $(\bmod F)$ of $k$ and corresponds via class field theory to a totally real abelian extension $F$ of $k$.

We define the character of $\operatorname{sign} \lambda(\alpha)$ on $k$ by

$$
\lambda(\alpha)=\prod_{j=2}^{n} \operatorname{sgn}\left(\alpha^{(j)}\right) .
$$

Let $\mathcal{F}_{0}$ denote the subgroup of all $(\alpha)$ in $S(F)$ such that $\lambda(\alpha)=1$ and $\mathcal{S}$ the set of all $(\alpha)$ in $S(F)$ such that $\lambda(\alpha)=-1$. It can happen that $S_{0}=\mathfrak{F}=S(F)$. The condition that this not occur is that for all units $\epsilon$ of $k$ congruent to 1 $(\bmod F)$, we must have $\lambda(\epsilon)=1$. We assume that $F$ satisfies this condition, and let $G=I(F) / \mathbb{S}_{0}$. By class field theory, $G$ corresponds to a real abelian extension $K$ of $k$ which is a quadratic extension of $F$.

For any $\mathbb{S}$ in $G$, let

$$
\zeta(s, \mathfrak{s})=\sum_{\mathcal{U} \in \mathfrak{S}} N(\mathfrak{Q})^{-s}
$$

where the sum is over all integral ideals $\mathfrak{l}$ of $\mathbb{E}$. Let

$$
\epsilon(\Im)=\exp \left[2 \zeta^{\prime}(0,(\xi)], \quad \epsilon=\epsilon\left(\xi_{0}\right) .\right.
$$

CONJECTURE 1. The numbers $\epsilon(\mathcal{S})$ are conjugate algebraic integers in $K$. If $\mathfrak{p}$ is a first degree prime ideal in $(\mathbb{S}$ of norm $p$ then the explicit reciprocity law of class field theory is given by

$$
\epsilon^{p} \equiv \epsilon(\mathcal{E})(\bmod \mathfrak{p})
$$

Our conjecture thus provides an answer to Hilbert's twelfth problem for totally real fields $k$. The purpose of this note is to present the first numerical example of Conjecture 1 with a nonabelian ground field $k$. Conjecture 1 implies that $\epsilon(\mathcal{S})=\epsilon(\mathbb{S})^{-1}$ is a unit, that

AMS (MOS) subject classifications (1970). Primary 12A65, 12 A70.

${ }^{1}$ Supported by NSF Grant MSC 7608184. 


$$
\alpha\left((5)=\alpha(5 \varsubsetneqq)=\epsilon(\Im)+\epsilon(\varsigma)^{-1}\right.
$$

is in $F$ and that

$$
g(x)=\prod_{\sqrt{ }\left(\bmod \mathfrak{S}_{0}, \mathfrak{I}\right)}(x-\alpha(\mathfrak{s}))=\sum_{j=0}^{|H|}(-1)^{j_{j}} x^{|H|-j}
$$

has coefficients in $k=k^{(1)}$.

We take $k=k^{(1)}=Q\left(\beta^{(1)}\right)$ where

$$
\beta=\beta^{(1)}=3.07911886452947847 \cdots
$$

is one of the three real roots of

$$
x^{3}-x^{2}-9 x+8=0 .
$$

The field $k$ has class-number 3, discriminant $2597=7^{2} \cdot 53\left(1, \beta, \beta^{2}\right.$ form an integral basis) and every unit $\epsilon$ of $k$ has $\lambda(\epsilon)=1$. Thus we may take $f=(1)$; $F$ is then the Hilbert class field of $k$ and $K$ is a sixth degree extension of $k$ which is a quadratic extension of $F$. The group $G$ is cyclic of order 6 and is generated by the element $\mathbb{E}_{1}$ containing the unique prime ideal $\mathcal{F}_{2}$ in $k$ of norm 2 . We let $\mathfrak{S}_{j}=\mathfrak{S}_{1}^{j}, 0 \leqslant j \leqslant 5$. In particular $\mathfrak{T}=\mathfrak{S}_{3}$. (Indeed $\mathfrak{p}_{2}^{3}=(\beta)$ and $\lambda(\beta)=-1$.)

The following values of $\zeta^{\prime}(0, \mathcal{\varsigma})$ were found on a computer which worked internally with an accuracy of about 16 decimal places:

$$
\begin{aligned}
& 2 \zeta^{\prime}\left(0, \mathfrak{\complement}_{0}\right)=2.6229258798145494=-2 \zeta^{\prime}\left(0, \mathfrak{S}_{3}\right) \text {, } \\
& 2 \zeta^{\prime}\left(0, \varsigma_{2}\right)=-.72668091960461237=-2 \zeta^{\prime}\left(0, \varsigma_{5}\right) \text {, } \\
& 2 \xi^{\prime}\left(0, \aleph_{4}\right)=-.55674277199362199=-2 \zeta^{\prime}\left(0, \mathcal{\mho}_{1}\right) \text {. }
\end{aligned}
$$

We put $\epsilon_{j}=\epsilon\left(\mathbb{S}_{j}\right), \alpha_{j}=\alpha\left(\mathbb{S}_{j}\right)$. We then get

$$
\begin{aligned}
g(x)= & \left(x-\alpha_{0}\right)\left(x-\alpha_{2}\right)\left(x-\alpha_{4}\right) \\
= & x^{3}-18.718329575489666 x^{2}+73.354291283859894 x \\
& -81.914383130290574 .
\end{aligned}
$$

The coefficients of $g(x)$ are supposed to be in $k=k^{(1)}$ (in other words, we are getting a particular embedding of $k$ out of Conjecture 1 as well as a particular embedding of $K$ and $F)$. Conjecture 1 yields bounds on $\theta_{j}^{(i)}(i=2,3)$ and so leads us to the numbers

$$
\begin{aligned}
\beta^{2}+3 \beta & =18.718329575489740, \\
5 \beta^{2}+12 \beta-11 & =73.354291283860260, \\
6 \beta^{2}+13 \beta-15 & =81.914383130291046,
\end{aligned}
$$

which must be $\theta_{1}, \theta_{2}$ and $\theta_{3}$ respectively if Conjecture 1 holds.

It may be checked that any root $A$ of 


$$
x^{3}-\left(\beta^{2}+3 \beta\right) x^{2}+\left(5 \beta^{2}+12 \beta-11\right) x-\left(6 \beta^{2}+13 \beta-15\right)=0
$$

does indeed generate $F$ and that either root $E$ of $x+x^{-1}=A$ is a unit in $K$ which in fact generates $K$ over $Q$. Lastly, the reciprocity law is as given by Conjecture 1 .

Let $\epsilon^{\prime}=\epsilon_{0} \epsilon_{2} \epsilon_{4}$. Conjecture 1 implies that $\epsilon^{\prime}$ is the relative norm of $\epsilon$ from $K$ to a quadratic extension $K^{\prime}$ of $k$. We have shown without assuming Conjecture 1 that $\epsilon^{\prime}$ generates the unique quadratic extension of $k$ lying in $K$ and that

$$
\epsilon^{\prime}+\left(\epsilon^{\prime}\right)^{-1}=\beta+1 \text {. }
$$

This serves both as a check on the reciprocity part of Conjecture 1 and on the accuracy of the computation of the numbers $\xi^{\prime}(0,(\Sigma)$.

Some comments about the actual computation may be useful. The function

$$
\left(\frac{2597}{(2 \pi)^{3}}\right)^{s / 2} \Gamma\left(\frac{s}{2}\right) \Gamma\left(\frac{s+1}{2}\right)^{2}[\zeta(s,(s)-\zeta(s,(\varsigma))]
$$

is given by a triple integral of a three-dimensional $\theta$-function and we are interested in the value of this integral at $s=0$. The triple integral splits into two pieces via the inversion formula for $\theta$-functions. At $s=0$, one of these pieces splits into an infinite sum of single integrals of the form

$$
I_{1}(a)=\int_{0}^{\infty} \exp \left[-a\left(x+2 x^{-1 / 2}\right)\right] d x
$$

while the other piece splits into an infinite sum of double integrals of the form

$$
I_{2}(a)=\int_{0}^{\infty} \int_{a}^{\infty}(x t)^{-1 / 2} \exp \left[-t\left(x+2 x^{-1 / 2}\right)\right] d t d x .
$$

The interior integral in $I_{2}$ for a given $x$ was integrated using the continued fraction expansion of the incomplete gamma function as analyzed by $\mathrm{R}$. Terras [2]. The integral over $x$ in $I_{2}$ was then computed numerically as was the integral for $I_{1}$. Several hundred integrals of each type were required in the computation. In the procedure finally used, the field $K$ cost $\$ 7$. Still, it would be very worthwhile for future computations to have a rapid accurate algorithm for computing $I_{1}$ and $I_{2}$ for a wide range of $a$.

More details regarding this example, examples with real quadratic $k$ and analogies with complex quadratic $k$ will be found in [1]

\section{REFERENCES}

1. H. M. Stark, L-functions at $s=1$. III. Totally real fields and Hilbert's twelfth problem, Advances in Math. 22 (1976), 64-84. IV (to appear).

2. Riho Terras, On the convergence of the continued fraction for the incomplete gamma function and an algorithm for the Riemann zeta function (to appear).

DEPARTMENT OF MATHEMATICS, MASSACHUSETTS INSTITUTE OF TECHNOLOGY, CAMBRIDGE, MASSACHUSETTS 02139 